\title{
Constraints on ultralight axions from compact binary systems
}

\author{
Tanmay Kumar Poddar $\odot^{1,{ }^{*}}$ Subhendra Mohanty, ${ }^{1, \dagger}$ and Soumya Jana $\odot^{1,2, \$}$ \\ ${ }^{1}$ Theoretical Physics Division, Physical Research Laboratory, Ahmedabad 380009, India \\ ${ }^{2}$ Département de Physique Théorique, Université de Genève, \\ 24 quai Ernest Ansermet, 1211 Genève 4, Switzerland
}

(Received 12 October 2019; accepted 19 March 2020; published 7 April 2020)

\begin{abstract}
Ultralight particles $\left(m_{a} \sim 10^{-21}-10^{-22} \mathrm{eV}\right)$ with axionlike couplings to other particles can be candidates for fuzzy dark matter (FDM) if the axion decay constant $f_{a} \sim 10^{17} \mathrm{GeV}$. If a compact star is immersed in such a low-mass axionic potential, it develops a long-range field outside the star. This axionic field is radiated away when the star is in a binary orbit. The orbital period of a compact binary decays mainly due to the gravitational wave radiation, which was confirmed first in the Hulse-Taylor binary pulsar. The orbital period can also decay by radiation of other light particles like axions and axionlike particles (ALPs). For axionic radiation to take place, the orbital frequency of the periodic motion of the binary system should be greater than the mass of the scalar particle which can be radiated. This implies that, for most of the observed binaries, particles with mass $m_{a}<10^{-19} \mathrm{eV}$ can be radiated, which includes FDM particles. In this paper, we consider four compact binary systems-PSR J0348 + 0432, PSR J0737 - 3039, PSR J1738 + 0333, and PSR B1913 + 16 (Hulse-Taylor binary) - and show that the observations of the decay in orbital period set a bound on the axion decay constant of $f_{a} \lesssim \mathcal{O}\left(10^{11} \mathrm{GeV}\right)$. This implies that fuzzy dark matter cannot couple to gluons.
\end{abstract}

DOI: 10.1103/PhysRevD.101.083007

\section{INTRODUCTION}

The axion was first introduced to solve the strong $C P$ problem [1-4]. The most stringent probe of the strong $C P$ violation is the electric dipole moment of the neutron. Quantum chromodynamics (QCD) is one of the possible theories which can explain the strong interaction. However, the theory has a problem known as the strong $C P$ problem. We can write the QCD Lagrangian

$$
\begin{aligned}
\mathcal{L}= & -\frac{1}{4} G_{\mu \nu}^{a} G^{a \mu \nu}+\sum_{i=1}^{n}\left[\bar{q}_{i} i D q_{i}-\left(m_{i} q_{L i}^{\dagger} q_{R i}+\text { H.c. }\right)\right] \\
& +\theta \frac{g_{s}^{2}}{32 \pi^{2}} G_{\mu \nu}^{a} \tilde{G}^{a \mu \nu},
\end{aligned}
$$

where the dual of the gluon field strength tensor is

$$
\tilde{G}^{\mu \nu}=\frac{1}{2} \epsilon^{\mu \nu \gamma \delta} G_{\gamma \delta} .
$$

\footnotetext{
*tanmay@prl.res.in

†mohanty@prl.res.in

†Soumya.Jana@etu.unige.ch
}

Published by the American Physical Society under the terms of the Creative Commons Attribution 4.0 International license. Further distribution of this work must maintain attribution to the author(s) and the published article's title, journal citation, and DOI. Funded by SCOAP.
The last term in the QCD Lagrangian violates the discrete symmetries $P, T, C P$. Since all the quark masses are nonzero, the $\theta$ term in the Lagrangian must be present. The QCD depends on $\theta$ through some combination of parameters, $\bar{\theta}=\theta+\arg (\operatorname{det}(M))$, where $M$ is the quark mass matrix $[5,6]$. The neutron electric dipole moment (EDM) depends on $\bar{\theta}$, and from chiral perturbation theory we can obtain the neutron EDM as $d_{n} \simeq$ few $\times 10^{-16} \bar{\theta}$ e.cm. However, the current experimental constraint on the neutron EDM is $d_{n}<$ few $\times 10^{-26}$ e.cm, which implies $\bar{\theta} \lesssim$ $10^{-10}$ [7]. The smallness of $\bar{\theta}$ is called the strong $C P$ problem. To solve this, Peccei and Quinn, in 1977 [1], came up with the idea that $\bar{\theta}$ is not just a parameter but a dynamical field driven to zero by its own classical potential. They postulated a global $U_{P Q}(1)$ quasisymmetry, which is a symmetry at the classical level, but explicitly broken by the nonperturbative QCD effects which produce the $\theta$ term, and spontaneously broken at a scale $f_{a}$. Thus, the pseudo-Nambu-Goldstone bosons appear, and these are known as the axions. The QCD axion mass $\left(m_{a}\right)$ is related to the axion decay constant $\left(f_{a}\right)$ by $m_{a}=$ $5.7 \times 10^{-12} \mathrm{eV}\left(\frac{10^{18} \mathrm{GeV}}{f_{a}}\right)$. So, if we need an axion decay constant less than the Planck scale $\left(M_{\mathrm{pl}}\right)$, then the mass of the axion is $m_{a} \gtrsim 10^{-12} \mathrm{eV}$ [8]. Also, there are other pseudoscalar particles which are not the actual QCD axions, but these particles have many similar properties to the QCD axions. These are called the axionlike particles 
(ALPs). For ALPs, the mass and decay constant are independent of each other. These ALPs are motivated from string theory [9]. The interaction of ALPs with the standard model particles is governed by the Lagrangian [10]

$$
\begin{aligned}
\mathcal{L}= & \frac{1}{2} \partial_{\mu} a \partial^{\mu} a-\frac{\alpha_{s}}{8 \pi} g_{a g} \frac{a}{f_{a}} G_{a}^{\mu \nu} \tilde{G}_{\mu \nu}^{a}-\frac{\alpha}{8 \pi} g_{a \gamma} \frac{a}{f_{a}} F^{\mu \nu} \tilde{F}_{\mu \nu} \\
& +\frac{1}{2} \frac{1}{f_{a}} g_{a f} \partial_{\mu} a \bar{f} \gamma^{\mu} \gamma_{5} f,
\end{aligned}
$$

where $g$ 's are the coupling constants, which depend on the model. The first term is the dynamical term of ALPs. The second, third, and last terms denote the coupling of ALPs with the gluons, photons, and fermion fields, respectively. ALPs couple with the SM particles very weakly because the couplings are suppressed by $\frac{1}{f_{a}}$, where $f_{a}$ is called the axion decay constant, and for ALPs, it generally takes a larger value.

There is no direct evidence of axions in the Universe. However, there are lots of experimental and astrophysical bounds on axion parameters. There are some ongoing searches for solar axions which correspond to $f_{a} \sim$ $10^{7} \mathrm{GeV}$ having sub-eV masses [11,12]. If solar axions were there, then it would violate the supernova 1987A result, which requires $f_{a} \gtrsim 10^{9} \mathrm{GeV}$. Axions with $f_{a} \lesssim 10^{8} \mathrm{GeV}$ provide the component of hot dark matter [13-15]. Large values of $f_{a}$ are allowed in the anthropic axion window and can be studied by isocurvature fluctuations [16]. The laboratory bounds for the axions are discussed in Refs. [17-24]. The cosmological bounds for the cold axions produced by the vacuum realignment mechanism are discussed in Refs. $[25,26]$. The bounds on the axion mass and decay constant are discussed in Refs. [27-29], if cold axions are produced by the decay of axion strings.

Explaining the nature of dark matter and dark energy is a major unsolved problem in modern cosmology. An interesting dark matter model is fuzzy dark matter (FDM) [30,31]. FDM is made up of axionlike particles (ALPs) with mass $\left(10^{-21}-10^{-22} \mathrm{eV}\right)$ such that the associated de Broglie wavelength is comparable to the size of a dwarf galaxy ( 2 kpc). Axions and ALPs can be possible dark matter candidates [32] or can be dynamical dark energy [33]. Axions can also form clouds around a black hole or neutron star from superradiance instabilities and change the mass and spin of the star $[34,35]$. Cold FDM can be produced by an initial vacuum misalignment and, to have the correct relic dark matter density, the axion decay constant should be $f_{a} \sim 10^{17} \mathrm{GeV}$ [31]. This ultralight FDM was introduced to solve the cuspy halo problem.

ALPs are pseudo-Nambu-Goldstone bosons which have a spin-dependent coupling with nucleons so that, in an unpolarized macroscopic body, there is no net long-range field for ALPs outside the body. However, if the ALPs also have a $C P$-violating coupling, then they can mediate longrange forces even in unpolarized bodies [36,37].
It has been pointed out recently [38] that if a compact star is immersed in an axionic potential (which will take place if the ALPs are FDM candidates), a long-range field is developed outside the star.

The ALPs can be sourced by compact binary systems such as neutron star-neutron star (NS-NS) or neutron starwhite dwarf (NS-WD) and can have very small mass $\left(<10^{-19} \mathrm{eV}\right)$. They can be possible candidates of FDM. The FDM density arises from the coherent oscillation of an axionic field in free space. If such axionic FDM particles have a coupling with nucleons, then the compact objects (NS, WD) immersed in the dark matter potential develop long-range axionic hair. When such compact stars are in a binary orbit, they can lose orbital period by radiating the axion hair in addition to the gravitational wave [38,39].

In this paper, we study a model of ALPs sourced by the compact stars and put bounds on $f_{a}$ from the observations of the orbital period decay of compact binaries.

The paper is organized as follows: In Sec. II, we compute in detail the axionic charge (including GR corrections) of compact stars immersed in a (ultra)low-mass axionic background potential. In Sec. III, we show how the axionic scalar Larmor radiation can change the orbital period of compact binary systems. There may also be an axionmediated long-ranged fifth force between the stars in a binary system. In Sec. IV, we put constraints on $f_{a}$ for four compact binaries, PSR J0348 + 0432 [40], PSR J0737 3039 [41], PSR J1738 + 0333 [42], and PSR B1913 + 16 (the Hulse-Taylor binary) $[39,43]$, available in the literature. In Sec. V, we discuss the implication of the ALPs sourced by the compact binaries as the FDM. Finally, we summarize our results.

We use the units $\hbar=c=1$ throughout the paper.

\section{THE AXION PROFILE FOR AN ISOLATED NEUTRON STAR/WHITE DWARF}

The axion Lagrangian at the leading order of $1 / f_{a}$ is given in Eq. (3). The axion pseudoshift symmetry $a \rightarrow$ $a+\delta$ is used to remove the QCD theta angle. Suppose the fermions are quarks and we give a chiral rotation to the quark field, so that only the nonderivative coupling appears through the quark mass term. Such a field redefinition allow us to move the nonderivative couplings into the two lightest quarks, and all other quarks are integrated out. So, we can work in the effective two-flavor theory. Thus, in the chiral expansion, all the nonderivative dependence of the axion is contained in the pion mass term of the Lagrangian

$$
\mathcal{L} \supset 2 B_{0} \frac{f_{\pi}^{2}}{4}\left\langle U M_{a}^{\dagger}+M_{a} U^{\dagger}\right\rangle,
$$

where $U=e^{\frac{i \Pi}{f \pi}}$ and $\Pi=\left[\begin{array}{cc}\pi^{0} & \sqrt{2} \pi^{+} \\ \sqrt{2} \pi^{-} & -\pi^{0}\end{array}\right]$, and $B_{0}$ is related with the chiral condensate and determined by the pion mass term. $f_{\pi}$ is called the pion decay constant. We can obtain 
the effective axion potential from the neutral pion sector. On the vacuum, the neutral pion attains a vacuum expectation value and can trivially be integrated out, leaving the effective potential [44]

$$
V \approx-m_{\pi}^{2} f_{\pi}^{2} \sqrt{1-\frac{4 m_{u} m_{d}}{\left(m_{u}+m_{d}\right)^{2}} \sin ^{2}\left(\frac{a}{2 f_{a}}\right)},
$$

where $m_{u}$ and $m_{d}$ are the up and down quark masses, respectively, and $m_{\pi}$ is the mass of the pion.

It has been pointed out in Ref. [38] that, if we consider ALPs which couple to nucleons, then compact stars such as neutron stars and white dwarfs can be the source of longrange axionic force. The reason for this long-range force is as follows. In the vacuum, the potential for the ALPs is

$$
V \approx-\epsilon m_{\pi}^{2} f_{\pi}^{2} \sqrt{1-\frac{4 m_{u} m_{d}}{\left(m_{u}+m_{d}\right)^{2}} \sin ^{2}\left(\frac{a}{2 f_{a}}\right)} .
$$

For simplicity, we choose $m_{u}=m_{d}$, and therefore, the mass of the ALPs in vacuum becomes

$$
m_{a}=\frac{m_{\pi} f_{\pi}}{2 f_{a}} \sqrt{\epsilon} .
$$

Inside a compact star, the quark masses are corrected by the nucleon density, and the potential inside the star changes to

$V=-m_{\pi}^{2} f_{\pi}^{2}\left\{\left(\epsilon-\frac{\sigma_{N} n_{N}}{m_{\pi}^{2} f_{\pi}^{2}}\right)\left|\cos \left(\frac{a}{2 f_{a}}\right)\right|+\mathcal{O}\left(\left(\frac{\sigma_{N} n_{N}}{m_{\pi}^{2} f_{\pi}^{2}}\right)^{2}\right)\right\}$

and

$$
\sigma_{N}=\sum_{q=u, d} m_{q} \frac{\partial m_{N}}{\partial m_{q}}
$$

where $n_{N}$ is the nucleon number density, $m_{q}$ is the quark mass, $m_{\pi}$ is the pion mass, and $f_{\pi}$ is the pion decay constant. $\sigma_{N} \sim 59 \mathrm{MeV}$ from lattice simulation [45], and we consider the parameter space where $\epsilon \leq 0.1$ [38]. The tachyonic mass of the ALPs is the square root of the second derivative of the potential [Eq. (8)] at $a=0$. Inside the neutron star, $\sigma_{N} n_{N} / m_{\pi}^{2} f_{\pi}^{2}$ is not equal to zero, and $m_{T} \gtrsim m_{a}$. Thus, the magnitude of the tachyonic mass of the ALPs inside the compact star becomes

$$
m_{T}=\frac{m_{\pi} f_{\pi}}{2 f_{a}} \sqrt{\frac{\sigma_{N} n_{N}}{m_{\pi}^{2} f_{\pi}^{2}}-\epsilon}, \quad r<r_{\mathrm{NS}},
$$

where $r_{\mathrm{NS}}$ is the radius of the compact star. The compact star can be the source of ALPs if its size is larger than the critical size given by [38]

$$
r_{c} \gtrsim \frac{1}{m_{T}}
$$

For a typical neutron star and white dwarf, the condition in Eq. (11) is satisfied. By matching the axionic field solutions inside and outside the compact star, we get the long-range behavior of the axionic field. The axionic potential has degenerate vacua, and this degeneracy can be weakly broken by higher-dimensional operators suppressed by the Planck scale [46]. The degeneracy can also be broken by a finite-density effect like the presence of a NS and WD. At very high nuclear density, the axionic potential changes its sign, which allows the ALPs to be sourced by compact stars. Due to the very small size of the nuclei, it cannot be the source of the ALPs, and long-range axion fields arise only in large-sized objects like NSs and WDs.

Using Eq. (7) in Eq. (10), we can write the tachyonic mass as

$$
m_{T}^{2}=\sigma_{N} n_{N} / 4 f_{a}^{2}-m_{a}^{2} .
$$

Using the values of all the parameters and $m_{a} \sim 10^{-19} \mathrm{eV}$, we get the upper bound of the axion decay constant [using Eq. (11)] as $f_{a} \lesssim 2.636 \times 10^{17} \mathrm{GeV}$. Axions can never be sourced by a neutron star if $f_{a}$ is greater than this upper bound. Similarly, a white dwarf cannot be the source of axions if $f_{a} \gtrsim 9.95 \times 10^{14} \mathrm{GeV}$.

Compact stars with a large nucleon number density can significantly affect the axion potential. The second derivative of the potential [Eq. (8)] with respect to the field value is

$\frac{\partial^{2} V}{\partial a^{2}}=m_{\pi}^{2} f_{\pi}^{2}\left\{\left(\epsilon-\frac{\sigma_{N} n_{N}}{m_{\pi}^{2} f_{\pi}^{2}}\right) \frac{1}{4 f_{a}^{2}} \cos \left(\frac{a}{2 f_{a}}\right)+\mathcal{O}\left(\left(\frac{\sigma_{N} n_{N}}{m_{\pi}^{2} f_{\pi}^{2}}\right)^{2}\right)\right\}$.

Outside of the compact star, $\sigma_{N}=0$, which implies that

$\frac{\partial^{2} V}{\partial a^{2}}=m_{\pi}^{2} f_{\pi}^{2}\left\{\epsilon \frac{1}{4 f_{a}^{2}} \cos \left(\frac{a}{2 f_{a}}\right)+\mathcal{O}\left(\left(\frac{\sigma_{N} n_{N}}{m_{\pi}^{2} f_{\pi}^{2}}\right)^{2}\right)\right\}$.

Therefore, outside of the compact star $\left(r>r_{\mathrm{NS}}\right)$, the potential attains minima $\left(\frac{\partial^{2} V}{\partial a^{2}}>0\right)$ corresponding to the field values $a=0, \pm 4 \pi f_{a}, \ldots$ and maxima $\left(\frac{\partial^{2} V}{\partial a^{2}}<0\right)$ corresponding to the field values $a= \pm 2 \pi f_{a}, \pm 6 \pi f_{a} \ldots$, etc.

Inside the compact star $\left(r<r_{\mathrm{NS}}\right), \sigma_{N} \neq 0$ and $\frac{\sigma_{N} n_{N}}{m_{\pi}^{2} f_{\pi}^{2}}>\epsilon$. Therefore, inside the compact star, the potential has maxima at $a=0, \pm 4 \pi f_{a}, \ldots$ and minima at the field values $a= \pm 2 \pi f_{a}, \pm 6 \pi f_{a} \ldots$, etc.

The axionic field becomes tachyonic inside of a compact star and resides on one of the local maxima of the axionic potential; outside of the star, the axionic field rolls down to the nearest local minimum and stabilizes about it. The axionic field asymptotically reaches zero $(a=0)$ at 
infinity. Therefore, throughout the interior of the compact star, the axionic field assumes a constant value $a=4 \pi f_{a}$, the nearest local maximum.

For an isolated compact star of constant density, the equation of motion for the axionic field is [38]

$\nabla^{\mu} \nabla_{\mu}\left(\frac{\theta}{2}\right)= \begin{cases}-m_{T}^{2} \sin \left(\frac{\theta}{2}\right) \operatorname{sgn}\left\{\cos \left(\frac{\theta}{2}\right)\right\} & \left(r<r_{\mathrm{NS}}\right), \\ m_{a}^{2} \sin \left(\frac{\theta}{2}\right) \operatorname{sgn}\left\{\cos \left(\frac{\theta}{2}\right)\right\} & \left(r>r_{\mathrm{NS}}\right),\end{cases}$

where $\theta=a / f_{a}$. The sgn function is required to take care of the absolute value $|\cos (\theta / 2)|$ in the potential. Note that the equation of motion for the axionic field inside the compact star is satisfied by the field value $a=4 \pi f_{a}$.

Assuming the exterior spacetime geometry due to the compact star to be the Schwarzschild, the axionic field equation [Eq. (15)] becomes

$$
\left(1-\frac{2 G M}{r}\right) \frac{d^{2} a}{d r^{2}}+\frac{2}{r}\left(1-\frac{G M}{r}\right) \frac{d a}{d r}=m_{a}^{2} a,
$$

where $M$ is the mass of the compact star, $G$ is Newton's gravitational constant, and we have used the approximation $\sin (\theta / 2) \approx \theta / 2$ for small $\theta$.

At a large distance $(r \gg 2 G M)$ from the compact star, the axionic field [Eq. (16)] becomes

$$
\frac{d^{2} a}{d r^{2}}+\frac{2}{r} \frac{d a}{d r}=m_{a}^{2} a
$$

Assuming $a=\xi(r) / r$, the above equation reduces to $\xi^{\prime \prime}-m_{a}^{2} \xi=0$ (where the prime denotes a derivative with respect to $r$ ). This has the solution $\xi=C_{1} e^{m_{a} r}+C_{2} e^{-m_{a} r}$. Since $a \rightarrow 0$ in the limit $r \rightarrow \infty, C_{1}=0$. Thus, $a$ behaves as $a \sim q_{\mathrm{eff}} e^{-m_{a} r} / r$, where we rename the integration constant $C_{2}$ as $q_{\text {eff }}$. Further, for sufficiently light mass $\left(m_{a} \ll 1 / D \ll 1 / r_{\mathrm{NS}}\right.$, where $D$ is the distance between the stars in a binary system), the scalar field has a longrange behavior with an effective charge $q_{\text {eff }}$. For scalar Larmor radiation, the orbital frequency $(\omega)$ of the binary pulsar should be greater than the mass of the particle that is radiated (i.e., $\omega>m_{a}$ ). This translates the mass spectrum of radiated ALPs for a typical neutron star-neutron star (NS-NS) or neutron star-white dwarf (NS-WD) binary system into $m_{a} \lesssim 10^{-19} \mathrm{eV}$. Also, the axion Compton wavelength should be much larger than the binary distance in order to use the massless limit in the computation of scalar radiation and effective charge, i.e., $m_{a}^{-1} \gg D$. The critical value of axion mass required for the scalar radiation and the binary distance for four compact binary systems are given in Table I, which is consistent with the assumption of $m_{a} \lesssim 10^{-19} \mathrm{eV}$. Consequently, the axion Compton wavelength (inverse of axion mass) is larger than the binary distance, and hence, the size of the star (the size of a NS is $10^{20} \mathrm{GeV}^{-1}$, and the size of a WD is $10^{23} \mathrm{GeV}^{-1}$ ).

To identify the effective charge $q_{\text {eff }}$, we exploit the continuity of the axion field across the surface of the
TABLE I. Summary of the axion Compton wavelength $\left(m_{a}^{-1}\right)$ and binary distance $D$ for all four compact binaries. All relevant parameters for the numerical calculation are given in Sec. IV.

\begin{tabular}{lcc}
\hline \hline Binary system & $\begin{array}{c}\text { Critical value of } \\
m_{a}^{-1}\left(\mathrm{GeV}^{-1}\right)\end{array}$ & $\begin{array}{c}\text { Binary separation } \\
D\left(\mathrm{GeV}^{-1}\right)\end{array}$ \\
\hline PSR J0348 + 0432 & $2.14 \times 10^{27}$ & $4.64 \times 10^{24}$ \\
PSR J0737 - 3039 & $2.08 \times 10^{27}$ & $4.83 \times 10^{24}$ \\
PSR J1738 + 0333 & $7.41 \times 10^{27}$ & $9.65 \times 10^{24}$ \\
PSR B1913 + 16 & $6.76 \times 10^{27}$ & $1.08 \times 10^{25}$ \\
\hline \hline
\end{tabular}

compact star. Therefore, we solve Eq. (16) in the massless limit $\left(m_{a} \rightarrow 0\right)$, i.e.,

$$
\left(1-\frac{2 G M}{r}\right) \frac{d^{2} a}{d r^{2}}+\frac{2}{r}\left(1-\frac{G M}{r}\right) \frac{d a}{d r}=0 .
$$

Integrating Eq. (18), we get $a^{\prime}=-C_{3} / r^{2}(1-2 G M / r)$, and further integration yields $a=-\frac{C_{3}}{2 G M} \ln (1-2 G M / r)+C_{4}$, where $C_{3}$ and $C_{4}$ are integration constants. For the $r \gg$ $2 G M$ limit, $a \rightarrow q_{\mathrm{eff}} / r$, and therefore, $C_{3}=q_{\mathrm{eff}}$ and $C_{4}=0$. Therefore, we get the axionic field profile outside the compact star

$$
a=-\frac{q_{\mathrm{eff}}}{2 G M} \ln \left(1-\frac{2 G M}{r}\right)
$$

The behavior of the axionic potential as a function of the axionic field and distance are illustrated in Fig. 1. The nature of the axionic field as we go from the inside to the outside of a compact star is also shown in Fig. 1. The variation of the effective-charge-to-mass ratio of a compact star is shown in Fig. 2 as a function of the mass-to-radius ratio for different decay constants.

At the surface of the compact star, $a\left(r_{\mathrm{NS}}\right)=4 \pi f_{a}$. Thus, we identify

$$
q_{\mathrm{eff}}=-\frac{8 \pi G M f_{a}}{\ln \left(1-\frac{2 G M}{r_{\mathrm{NS}}}\right)}
$$

If $\frac{G M}{r_{\mathrm{NS}}} \ll 1, q_{\mathrm{eff}} \sim 4 \pi f_{a} r_{\mathrm{NS}}$ [38]. However, for a typical neutron star $\left(M=1.4 M_{\odot}\right.$ and $\left.r_{\mathrm{NS}}=10 \mathrm{~km}\right)$, the above correction is not negligible. For a white dwarf, the effect is negligible. The charges can both be positive as well as negative, depending on the sign of the axionic field values at the surface of the compact star. If $q_{1}$ and $q_{2}$ are the charges of two compact stars, then if $q_{1} q_{2}>0$, the two stars attract each other, and if $q_{1} q_{2}<0$, they repel each other [38]. For a neutron star, the new effective axion charge [Eq. (20)] is smaller than $4 \pi f_{a} r_{\mathrm{NS}}$ by $21.46 \%$. The effect of the new axion charge is illustrated in Fig. 3, where a plot of the axion profile both inside and outside a neutron star is shown. 


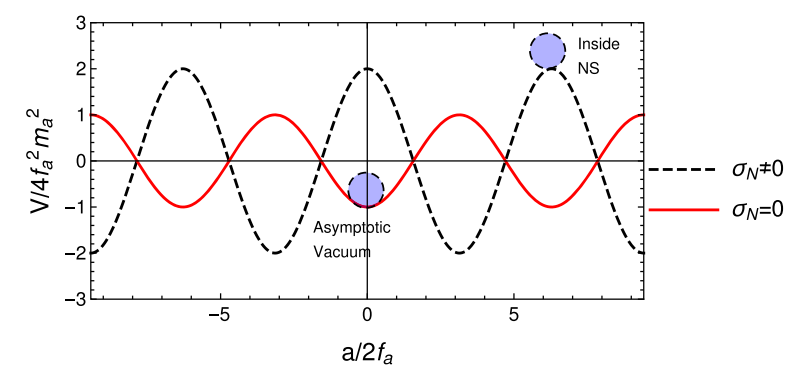

(a) $V$ vs. $a$

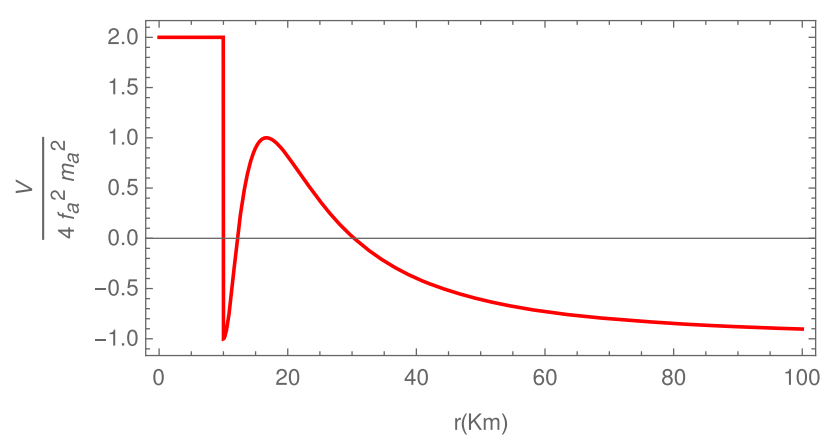

(b) $V$ vs. $r$

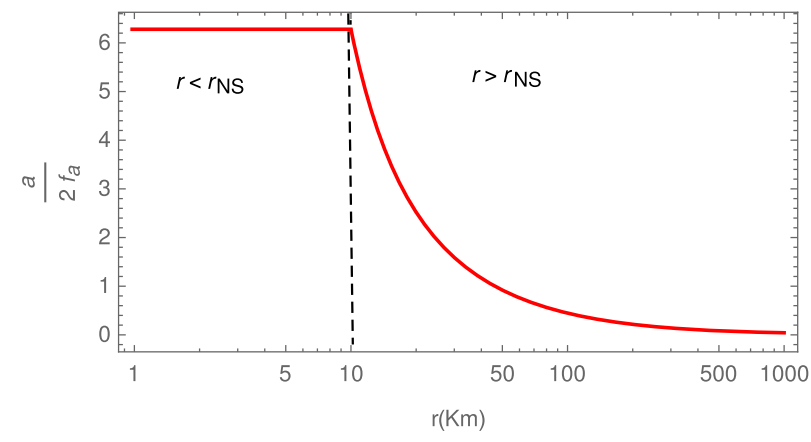

(c) $a$ vs. $r$

FIG. 1. (a) Plot of the axionic potential $V$ as a function of the axionic field. We assume $m_{T}^{2} / m_{a}^{2}=2$. The black dashed line corresponds to $\sigma_{N} \neq 0$ (i.e., inside the compact star), and the red solid line corresponds to $\sigma_{N}=0$. Note that the axionic field evolves from the local maximum $a=4 \pi f_{a}$ inside a compact star to the nearest local minimum $a=0$ outside the compact star. (b) The plot of $V$ as a function of $r$ inside and outside the neutron star. Note that there is discontinuity in $V(r)$ at $r=r_{\mathrm{NS}}$ due to sign change in the potential. (c) Plot of the axionic field $a$ as a function of $r$. We assume a neutron star as an example of the compact object in the plots. The typical mass and radius of a neutron star are $M=1.4 M_{\odot}$ and $r_{\mathrm{NS}}=10 \mathrm{~km}$, respectively. We can obtain a similar type of profile for white dwarfs.

\section{AXIONIC FIFTH FORCE AND THE SCALAR RADIATION FOR THE COMPACT BINARIES}

Such a long range axionic field mediates a "fifth" force (in addition to the Newtonian gravitational force) between the stars of a binary system (NS-NS or NS-WD),

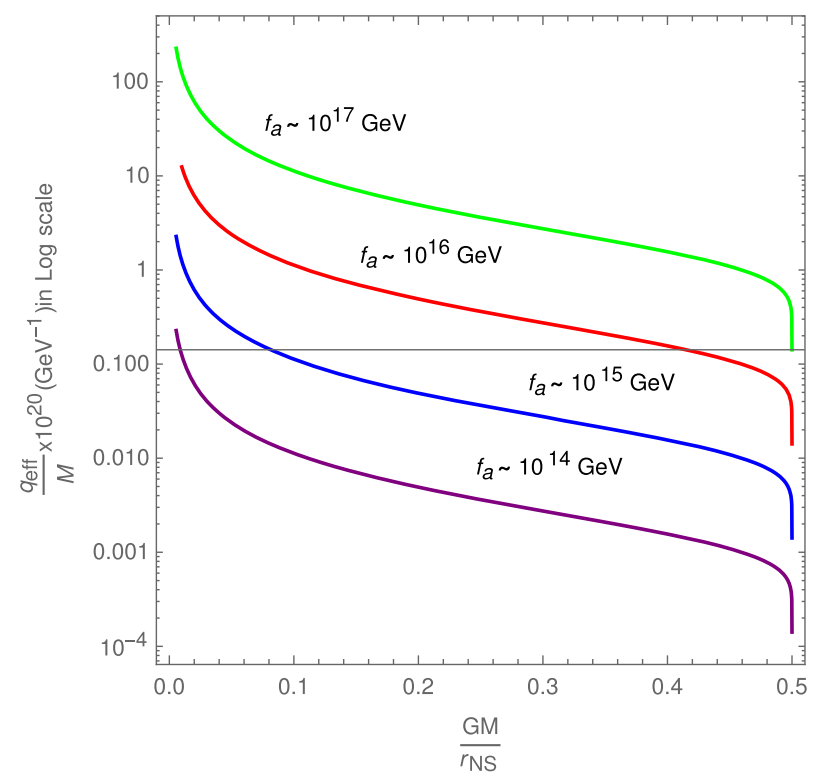

FIG. 2. The variation of the effective-charge-to-mass ratio of the neutron star with the ratio of mass to radius for different values of the axion decay constant.

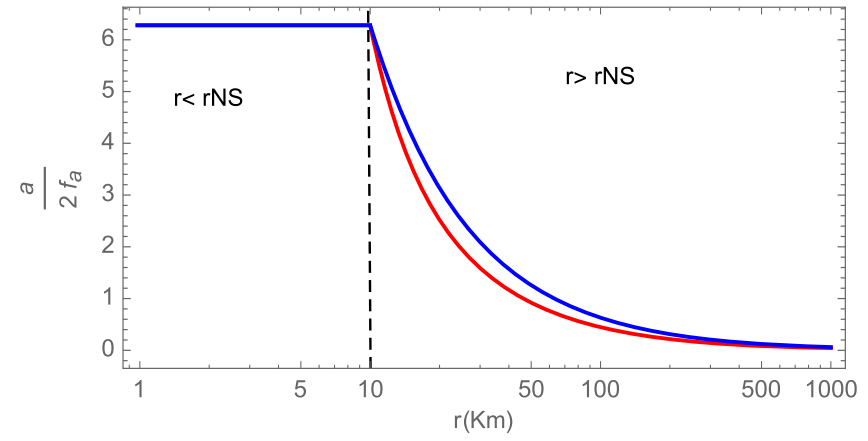

FIG. 3. Plot of the axion field $a$ as a function of $r$. The blue curve stands for the axion field $a \sim q_{\text {eff }} / r$, and the red curve stands for the axion field $a \sim-q_{\text {eff }} / 2 G M \ln (1-2 G M / r)$ outside the neutron star. For the blue curve, the effective axion charge is $q_{\mathrm{eff}}=4 \pi f_{a} r_{\mathrm{NS}}$, and for the red curve $q_{\mathrm{eff}}$ is given by Eq. (20).

$$
F_{5}=\frac{q_{1} q_{2}}{4 \pi D^{2}}
$$

where $q_{1,2}$ are effective charges of the stars in the binary system. Due to the presence of this scalar-mediated fifth force, Kepler's law is modified by [47]

$$
\omega^{2}=\frac{G\left(m_{1}+m_{2}\right)}{D^{3}}(1+\alpha),
$$

where $\alpha=\frac{q_{1} q_{2}}{4 \pi G m_{1} m_{2}}$ is the ratio of the scalar-mediated fifth force to the gravitational force, $\omega$ is the angular frequency of orbital motion of the stars, $m_{1}$ and $m_{2}$ are the masses of the stars, and $\mu=m_{1} m_{2} /\left(m_{1}+m_{2}\right)$ is the reduced mass of 
the binary system. There are constraints on the fifth force from either scalar-tensor theories of gravity [47-49] or the dark matter components [49-51]. In this paper, we show that the constraint on $\alpha$ from orbital period decay by scalar radiation is more stringent than the measured change in orbital period [Eq. (22)] due to fifth force.

The orbital period of the binary star system decays with time because of the energy loss, primarily due to the gravitational quadrupole radiation and about $1 \%$ due to ultralight scalar or pseudoscalar Larmor radiation. The total power radiated for such quasiperiodic motion of a binary system is

$$
\begin{aligned}
\frac{d E}{d t}= & -\frac{32}{5} G \mu^{2} D^{4} \omega^{6}\left(1-e^{2}\right)^{-\frac{7}{2}}\left(1+\frac{73}{24} e^{2}+\frac{37}{96} e^{4}\right) \\
& -\frac{\omega^{4} p^{2}}{24 \pi} \frac{\left(1+e^{2} / 2\right)}{\left(1-e^{2}\right)^{\frac{5}{2}}}
\end{aligned}
$$

where $e$ is the eccentricity of the elliptic orbit and $E$ is the total energy of the binary system. The first term on the rhs is the gravitational quadrupole radiation formula [39,50], and the second term is the massless scalar dipole radiation formula $[38,39,52]$. There is the radiation of the ALPs if the orbital frequency is greater than the mass of the ALPs. The dipole moment in the center-of-mass frame of the binary system can be written as

$$
p=q_{1} r_{1}-q_{2} r_{2}=q_{1} \frac{\mu D}{m_{1}}-q_{2} \frac{\mu D}{m_{2}}
$$

or

$$
p=8 \pi G f_{a} \mu D\left[\frac{1}{\ln \left(1-\frac{2 G m_{2}}{r_{\mathrm{NS}}}\right)}-\frac{1}{\ln \left(1-\frac{2 G m_{1}}{r_{\mathrm{NS}}}\right)}\right],
$$

where $r_{1,2}$ are the radial distances of the stars in the binary system from the center of mass along the semimajor axis. For nonzero scalar radiation, the charge-to-mass ratio $(\mathrm{q} / \mathrm{m})$ should be different for two stars. Thus, for the companion star in a binary system with the equal effective charge, there should be some mass difference of the two stars. The decay of the orbital time period is given by $[39,53]$

$\dot{P}_{b}=6 \pi G^{-\frac{3}{2}}(1+\alpha)^{-\frac{3}{2}}\left(m_{1} m_{2}\right)^{-1}\left(m_{1}+m_{2}\right)^{-\frac{1}{2}} D^{\frac{5}{2}}\left(\frac{d E}{d t}\right)$,

where $P_{b}=2 \pi / \omega$. NS-NS binaries (with different mass components) as well as NS-WD binaries are the sources for the scalar Larmor radiation and also for the axion-mediated fifth force. On the other hand, NS-BH systems can be the source of scalar radiation, but there is no long-range fifth force in between, as the scalar charges for the black holes (BHs) are zero [54].
In the next section, we consider four compact binaries and put constraints on $f_{a}$.

\section{CONSTRAINTS ON AXION PARAMETERS OF DIFFERENT COMPACT BINARIES}

\section{A. PSR J0348 + 0432}

This binary system consists of a neutron star and a lowmass white dwarf companion. The orbital period of the quasiperiodic binary motion is $P_{b}=2.46 \mathrm{~h}$. The mass of the neutron star in this binary system is $M_{p}=2.01 M_{\odot}$, and the mass of the white dwarf is $M_{\mathrm{WD}}=0.172 M_{\odot}$. The radius of the white dwarf is $r_{\mathrm{WD}}=0.065 R_{\odot}$, and we assume the radius of the neutron star is $r_{\mathrm{NS}}=10 \mathrm{~km}$. We compute the semimajor axis of the orbit using Kepler's law [Eq. (22)]. The observed decay of the orbital period is $\dot{P}_{b}=0.273 \times 10^{-12} \mathrm{~s} \mathrm{~s}^{-1}$ [40]. This is primarily due to gravitational quadrupole radiation from the binary NS-WD system. The contribution from the radiation of some scalar or pseudoscalar particles must be within the excess of the decay of the orbital period, i.e., $\dot{P}_{b \text { (scalar })} \leq\left|\dot{P}_{b(\text { observed })}-\dot{P}_{b(\mathrm{gw})}\right|$. If ALPs are emitted as scalar Larmor radiation, then we can find the upper bound on the axion decay constant. Using Eqs. (22), (23), (25), and (26) and taking the ALPs as massless, we obtain an upper bound on the axion decay constant as $f_{a} \lesssim 1.66 \times 10^{11} \mathrm{GeV}$. The ratio of the axionic fifth force and the Newtonian gravitational force between the stars in this system comes out to be $\alpha \lesssim 5.73 \times 10^{-10}$.

\section{B. PSR J0737 - 3039}

PSR J0737 - 3039 is a double neutron star binary system whose average orbital period is $P_{b}=2.4 \mathrm{~h}$. Its observed orbital period decays at a rate $\dot{P}_{b}=1.252 \times 10^{-12} \mathrm{~s} \mathrm{~s}^{-1}$. The pulsars have masses $M_{1}=1.338 M_{\odot}$ and $M_{2}=$ $1.250 M_{\odot}$. The eccentricity of the orbit is $e=0.088$ [41]. Using Eqs. (22), (23), (25), and (26), we obtain the upper bound on the axion decay constant as $f_{a} \lesssim 9.76 \times 10^{16} \mathrm{GeV}$. Besides the axion radiation, axion-mediated fifth force arises in this binary system. We obtain the value of $\alpha \lesssim 9.21 \times 10^{-3}$.

\section{PSR J1738+0333}

This pulsar-white dwarf binary system has an average orbital period $P_{b}=8.5 \mathrm{~h}$ and the orbit has a very low eccentricity, $e<3.4 \times 10^{-7}$. The mass of the pulsar is $M_{p}=1.46 M_{\odot}$, and the mass of the white dwarf is $M_{\mathrm{WD}}=$ $0.181 M_{\odot}$. The radius of the white dwarf is $r_{\mathrm{WD}}=$ $0.037 R_{\odot}$. The rate of the intrinsic orbital period decay is $\dot{P}_{b}=25.9 \times 10^{-15} \mathrm{~s} \mathrm{~s}^{-1}$ [42]. Using this system, we obtain the upper bound on the axion decay constant as $f_{a} \lesssim$ $2.03 \times 10^{11} \mathrm{GeV}$. The value of $\alpha$ comes out $\lesssim 8.59 \times 10^{-10}$. 


\section{PSR B1913 + 16: Hulse-Taylor binary pulsar}

The observed orbital period of the Hulse-Taylor binary decays at the rate of $\dot{P}_{b}=2.40 \times 10^{-12} \mathrm{~s} \mathrm{~s}^{-1}$. The masses of the stars in this binary system are $m_{1}=1.42 M_{\odot}$ and $m_{2}=$ $1.4 M_{\odot}$ [39]. The eccentricity of the orbit is $e=0.617127$, and the average orbital frequency is $\omega=0.2251 \times 10^{-3} \mathrm{~s}^{-1}$. For this system, we obtain the upper bound on the decay constant as $f_{a} \lesssim 2.12 \times 10^{17} \mathrm{GeV}$. We obtain the value of $\alpha$ for this system: $\lesssim 3.4 \times 10^{-2}$. Note that the binary orbit of this system is highly eccentric. As a result, the contributions of the eccentricity factors in the radiation formulas [Eq. (23)] are important. For the GW radiation, the eccentricity factor is 11.85 , and for the scalar radiation, it is 3.94 .

In Table II, we have obtained the upper bound of the axion decay constant and the relative strength of the axionmediated force for the four compact binaries.

\section{IMPLICATION FOR THE AXIONIC FUZZY DARK MATTER}

The ALPs that are radiated from the compact binaries can be possible candidates of FDM, whose mass is $\left.\sim \mathcal{O}\left(10^{-21}-10^{-22} \mathrm{eV}\right)\right)$. In the very early Universe, the axionic field evolves with a cosine potential

$$
V\left(\frac{a}{f_{a}}\right)=m_{a}^{2} f_{a}^{2}\left[1-\cos \left(\frac{a}{f_{a}}\right)\right]
$$

The equation of motion for the axionic field is

$$
\ddot{a}+3 H \dot{a}-\frac{1}{R^{2}} \nabla^{2} a+m_{a}^{2} a=0,
$$

where $R(t)$ is the scale factor in the FRW spacetime. Taking the Fourier transform of Eq. (28), the modes decouple, and we have

$$
\ddot{a}_{k}+3 H \dot{a}_{k}+\frac{k^{2}}{R^{2}} a_{k}+m_{a}^{2} a_{k}=0 .
$$

For nonrelativistic (small- $k$ ) or zero modes, the third term becomes zero, and the equation of motion of the axionic field is a damped harmonic oscillatory. The axionic field takes a constant value as long as $H \gtrsim m_{a}$, which fixes the

TABLE II. Summary of the upper bounds on the axion decay constant $f_{a}$ of ALPs radiated from compact binaries. For all the binaries, we assume $m_{a}<10^{-19} \mathrm{eV}$.

\begin{tabular}{lcc}
\hline \hline Compact binary system & $f_{a}(\mathrm{GeV})$ & $\alpha$ \\
\hline PSR J0348 + 0432 & $\lesssim 1.66 \times 10^{11}$ & $\lesssim 5.73 \times 10^{-10}$ \\
PSR J0737 - 3039 & $\lesssim 9.76 \times 10^{16}$ & $\lesssim 9.21 \times 10^{-3}$ \\
PSR J1738 + 0333 & $\lesssim 2.03 \times 10^{11}$ & $\lesssim 8.59 \times 10^{-10}$ \\
PSR B1913 + 16 & $\lesssim 2.12 \times 10^{17}$ & $\lesssim 3.4 \times 10^{-2}$ \\
\hline \hline
\end{tabular}

initial misalignment angle, and then the axionic field starts oscillating with a frequency $\sim m_{a}$. When the oscillation starts at $H \sim m_{a}$, then the energy density of axionic field is of the order of $m_{a}^{2} a_{0}^{2}$, where $a_{0}$ is the initial field value during inflation. The oscillation modes are damped as $R^{-\frac{3}{2}}$. The energy density of the axionic field, when it is oscillating, goes as $\frac{1}{R^{3}}$. Hence, at late times, the axionic energy density redshifts like a cold dark matter. The ratio of dark matter to radiation energy densities increases as $\frac{1}{T}$ with the expansion of the Universe, and the dark matter starts dominating over radiation at $T \sim 1 \mathrm{eV}$. Using these facts, the dark matter relic density becomes [31]

$$
\Omega_{\mathrm{DM}} \sim 0.1\left(\frac{a_{0}}{10^{17} \mathrm{GeV}}\right)^{2}\left(\frac{m_{a}}{10^{-22} \mathrm{eV}}\right)^{\frac{1}{2}}
$$

where $a_{0}=\theta_{0} f_{a}$, and $\theta_{0}$ is the initial misalignment angle which can take values in the range $-\pi<\theta_{0}<\pi$. Since the coupling of ALPs with matter is proportional to $\frac{1}{f_{a}}$, large values of $f_{a}$ correspond to weaker coupling with matter. Therefore, direct detection of the ALPs in this scale is much more difficult. However, the ALPs in this large- $f_{a}$ scale have some theoretical motivations [9]. The axion decay constant in the GUT scale implies that a single axion condensate can trigger the breaking of symmetries in nature. ALPs of mass $\mathcal{O}\left(10^{-21}-10^{-22} \mathrm{eV}\right)$ sourced by the binary systems can give rise to the correct relic density of FDM if the axion decay constant is $f_{a} \sim 10^{17} \mathrm{GeV}$ and the initial misalignment angle $\theta_{0} \sim \mathcal{O}(1)$. Any value of $f_{a}$ other than $10^{17} \mathrm{GeV}$ requires fine-tuning of the initial misalignment angle, which can take values from $-\pi$ to $+\pi$.

For the NS-WD binaries PSR J0348+0432 and PSR $\mathrm{J} 1738+0333$, the bound on the axion decay constant $\left[f_{a} \lesssim \mathcal{O}\left(10^{11} \mathrm{GeV}\right)\right]$ is well below the GUT scale, and this gives the stronger bound. This implies that if the ultralight ALPs have to be FDM, then they do not couple with gluons.

\section{CONCLUSIONS AND DISCUSSIONS}

In this paper, we have obtained upper bounds on the decay constant of the ultralight ALPs from the study of decay in orbital period of the compact binary stars (NS-NS, NS-WD). Compact stars such as neutron stars and white dwarfs can be the source of ALPs. We have assumed that the mass of the ALPs is sufficiently low such that the axionic field has a long-range behavior over a distance between the binary companions. Due to such an axionic field, the binary system will emit scalar Larmor radiation. Although the gravitational quadrupole radiation mainly contributes to the decay of orbital period, the contribution of scalar radiation is not negligible. However, its contribution must be within the excess value of the observed decay in the orbital period. For the NS-NS and NS-WD 
binary systems, an additional axionic "fifth" force arises which is not relevant as much as the scalar radiation in our study.

We have obtained the axionic profile for an isolated compact star, assuming it to be a spherical object of uniform mass density. We have identified the form of effective axionic charge of the compact star [38] and its GR correction. We have also considered eccentricity of the orbits of binary systems - a generalization of previous results for axionic scalar radiation [38]. Using the updated formula for the total power radiated, we have studied four compact binary systems: PSR J0348 + 0432, PSR J0737 - 3039, PSR J1738 + 0333, and PSR B1913 + 16 (the Hulse-Taylor binary pulsar). The upper bound on the axion decay constant $f_{a}$ is found as $f_{a} \lesssim \mathcal{O}\left(10^{11} \mathrm{GeV}\right)$.

If the masses of the ALPs which are sourced by compact binaries are $\mathcal{O}\left(10^{-21}-10^{-22} \mathrm{eV}\right)$ and $f_{a} \sim 10^{17} \mathrm{GeV}$, then they can contribute to the relic density of FDM. However, the bound $f_{a} \lesssim \mathcal{O}\left(10^{11} \mathrm{GeV}\right)$ from WD binaries does not favor ALPs as the FDM.
ALPs can give rise to isocurvature fluctuations during inflation which are tightly constrained from CMB observation. The Hubble scale during inflation (for single-field slow roll models) is $H_{I}=8 \times 10^{13} \sqrt{r / 0.1} \mathrm{GeV}$ [55] and therefore, for our bound $f_{a} \lesssim \mathcal{O}\left(10^{11} \mathrm{GeV}\right)$, it is possible to have $2 \pi f_{a}<H_{I}$, which means that the ALPs' symmetry breaking takes place after inflation, and there will be no isocurvature perturbations from ALPs. However, observations of Lyman- $\alpha$ disfavor FDM [56].

ALPs with a larger mass range $\left(m_{a}>10^{-19} \mathrm{eV}\right)$ can be probed from the observation of the gravitational wave signals from binary merger events at the LIGO-Virgo detectors. For this, detailed analysis of the gravitational waveform and phase is required which will take into account the energy loss by axionic emission.

\section{ACKNOWLEDGMENTS}

The authors thank Kent Yagi for suggesting a correction in the treatment of NS-WD binaries in this paper.
[1] R. D. Peccei and H. R. Quinn, Phys. Rev. Lett. 38, 1440 (1977).

[2] S. Weinberg, Phys. Rev. Lett. 40, 223 (1978).

[3] F. Wilczek, Phys. Rev. Lett. 40, 279 (1978).

[4] R. D. Peccei and H. R. Quinn, Phys. Rev. D 16, 1791 (1977).

[5] S. L. Adler, Phys. Rev. 177, 2426 (1969).

[6] J.S. Bell and R. Jackiw, Nuovo Cimento A 60, 47 (1969).

[7] C. A. Baker, D. D. Doyle, P. Geltenbort, K. Green, M. G. D. van der Grinten, P. G. Harris, P. Iaydjiev, S. N. Ivanov, D. J. R. May, J. M. Pendlebury, J. D. Richardson, D. Shiers, and K. F. Smith, Phys. Rev. Lett. 97, 131801 (2006).

[8] G. Grilli di Cortona, E. Hardy, J. Pardo Vega, and G. Villadoro, J. High Energy Phys. 01 (2016) 034.

[9] P. Svrcek and E. Witten, J. High Energy Phys. 06 (2006) 051.

[10] S. Profumo, An Introduction to Particle Dark Matter (World Scientific, Singapore, 2017).

[11] Y. Inoue, Y. Akimoto, R. Ohta, T. Mizumoto, A. Yamamoto, and M. Minowa, Phys. Lett. B 668, 93 (2008).

[12] E. Arik et al., J. Cosmol. Astropart. Phys. 02 (2009) 008;

[13] S. Hannestad, A. Mirizzi, and G. Raffelt, J. Cosmol. Astropart. Phys. 07 (2005) 002.

[14] A. Melchiorri, O. Mena, and A. Slosar, Phys. Rev. D 76, 041303(R) (2007).

[15] S. Hannestad, A. Mirizzi, G. G. Raffelt, and Y. Y. Y. Wong, J. Cosmol. Astropart. Phys. 04 (2008) 019.

[16] J. Hamann, S. Hannestad, G. G Raffelt, and Y. Y. Y. Wong, J. Cosmol. Astropart. Phys. 06 (2009) 022.

[17] Y. Semertzidis, R. Cameron, G. Cantatore, A. C. Melissinos, J. Rogers, H. Halama, A. Prodell, F. Nezrick, C. Rizzo, and E. Zavattini, Phys. Rev. Lett. 64, 2988 (1990).
[18] R.Cameron et al., Phys. Rev. D 47, 3707 (1993).

[19] C. Robilliard, R. Battesti, M. Fouche, J. Mauchain, A.-M. Sautivet, F. Amiranoff, and C. Rizzo, Phys. Rev. Lett. 99, 190403 (2007).

[20] A. S. Chou, W. Wester, A. Baumbaugh, H. R. Gustafson, Y. Irizarry-Valle, P. O. Mazur, J. H. Steffen, R. Tomlin, X. Yang, and J. Yoo, Phys. Rev. Lett. 100, 080402 (2008).

[21] P. Sikivie, D. B. Tanner, and K. van Bibber, Phys. Rev. Lett. 98, 172002 (2007).

[22] J. E Kim, Phys. Rep. 150, 1 (1987).

[23] H.-Y. Cheng, Phys. Rep. 158, 1 (1988).

[24] L. J. Rosenberg and K. A. van Bibber, Phys. Rep. 325, 1 (2000).

[25] M. P. Hertzberg, M. Tegmark, and F. Wilczek, Phys. Rev. D 78, 083507 (2008).

[26] L. Visinelli and P. Gondolo, Phys. Rev. D 80, 035024 (2009).

[27] R. A. Battye and E. P. S. Shellard, Phys. Rev. Lett. 73, 2954 (1994); 76, 2203(E) (1996).

[28] M. Yamaguchi, M. Kawasaki, and J. Yokoyama, Phys. Rev. Lett. 82, 4578 (1999).

[29] C. Hagmann, S. Chang, and P. Sikivie, Phys. Rev. D 63, 125018 (2001).

[30] W. Hu, R. Barkana, and A. Gruzinov, Phys. Rev. Lett. 85, 1158 (2000).

[31] L. Hui, J. P. Ostriker, S. Tremaine, and E. Witten, Phys. Rev. D 95, 043541 (2017).

[32] D. Duffy and K. van Bibber, New J. Phys. 11, 105008 (2009).

[33] M. Kamionkowski, J. Pradler, and D. G. E. Walker, Phys. Rev. Lett. 113, 251302 (2014). 
[34] D. Baumann, H. S. Chia, and R. A. Porto, Phys. Rev. D 99, 044001 (2019).

[35] F. V. Day and J. I. Mcdonald, J. Cosmol. Astropart. Phys. 10 (2019) 051.

[36] J.E. Moody and F. Wilczek, Phys. Rev. D 30, 130 (1984).

[37] G. Raffelt, Phys. Rev. D 86, 015001 (2012).

[38] A. Hook and J. Huang, J. High Energy Phys. 06 (2018) 036.

[39] S. Mohanty and P. K. Panda, Phys. Rev. D 53, 5723 (1996).

[40] J. Antoniadis et al., Science 340, 1233232 (2013).

[41] M. Kramer et al., Science 314, 97 (2006).

[42] P. C. C. Freire, N. Wex, G. Esposito-Farèse, J. P. W. Verbiest, M. Bailes, B. A. Jacoby, M. Kramer, I. H. Stairs, J. Antoniadis, and G. H. Janssen, Mon. Not. R. Astron. Soc. 423, 3328 (2012).

[43] R. A. Hulse and J. H. Taylor, Astrophys. J. 195, L51 (1975); J. H. Taylor and J. M. Weisberg, Astrophys. J. 253, 908 (1982); J. M. Weisberg and J. H. Taylor, Phys. Rev. Lett. 52, 1348 (1984).

[44] G. G. D. Cortona, E. Hardy, J. P. Vega, and G. Villadoro, J. High Energy Phys. 01 (2016) 034.
[45] J. M. Alarcon, J. M. Camalich, and J. A. Oller, Phys. Rev. D 85, 051503(R) (2012).

[46] M. Kamionkowsky and J. March-Russell, Phys. Lett. B 282, 137 (1992).

[47] S. Jana and S. Mohanty, Phys. Rev. D 99, 044056 (2019).

[48] T. Liu, X. Zhang, and W. Zhao, Phys. Lett. B 777, 286 (2018).

[49] S. Alexander, E. McDonough, R. Sims, and N. Yunes, Classical Quantum Gravity 35, 235012 (2018).

[50] D. Croon, A. E. Nelson, C. Sun, D. G. E. Walker, and Z.-Z. Xianyu, Astrophys. J. Lett. 858, L2 (2018).

[51] J. Kopp, R. Laha, T. Opferkuch, and W. Shepherd, J. High Energy Phys. 11 (2018) 096.

[52] D. E. Krause, H. T. Kloor, and E. Fischbach, Phys. Rev. D 49, 6892 (1994).

[53] P. C. Peters and J. Mathew, Phys. Rev. 131, 435 (1963).

[54] J. Huang, M. C. Johnson, L. Sagunski, M. Sakellariadou, and J. Zhang, Phys. Rev. D 99, 063013 (2019).

[55] K. Enqvist, R. J. Hardwick, T. Tenkanen, V. Vennin, and D. Wands, J. Cosmol. Astropart. Phys. 02 (2018) 006.

[56] T. Kobayashi, R. Murgia, A. De Simone, V. Irsic, and M. Viel, Phys. Rev. D 96, 123514 (2017). 